\title{
A comment on randomization checks in economics experiments
}

\author{
Sven Grüner (Corresponding Author) \\ Martin Luther University Halle-Wittenberg, Faculty of Natural Sciences III \\ Institute of Agricultural and Nutritional Sciences, Chair of Agribusiness Management \\ Karl-Freiherr-von-Fritsch-Str. 4, 06120 Halle (Saale), Germany \\ sven.gruener@landw.uni-halle.de
}

\begin{abstract}
This note deals with baseline comparisons in randomized controlled trials in economics. Although widely spread in the literature, resorting to $\mathrm{p}$-values is an inadequate procedure to assess whether randomization was successfully carried out. Instead, it is promising to use standardized differences. In addition, challenges to evaluate the quality of randomization appear if self-selection is systematically different across the compared treatments.
\end{abstract}

\section{Keywords}

Randomization check; p-value; self-selection

\section{JEL}

C90; C10

\section{Introduction}

In many economic experiments, randomized controlled trials (RCTs) are performed. In RCTs subjects are randomly assigned to either the control or treatment group(s). Under perfect conditions, randomization leads to balance of known and unknown covariates across all groups. Differences between treatment and control group can then be traced back to the intervention as intended by the experimenter (e.g. Borenstein 2009; Dunning 2012). However, randomization establishes blance of covariates across the groups only in expectation. In single runs of experiments or small-sized samples, we may worry about confounders which complicate identifying causal relationships, as it is prevalent in observational studies (Deaton and Cartwright 2018). This raises the question of how to assess whether the randomisation was successful or not. 


\section{Baseline comparisons: no p-values, but standard differences}

A large number of studies rely on significance tests in baseline comparisons. The literature provides evidence for statements such as "the variable is statistically significantly different in the two groups, therefore it should be added in regression analysis" or "the variable is not statistically significantly different in the two groups, therefore it should be assumed that there is no difference and there is no need to include it in the regression analysis." However, p-values are inappropriate to evaluate the success of randomization. ${ }^{1}$ Let us remind ourselves of the definition of p-values: "The $P$ value is [...] the probability that the chosen test statistic would have been at least as large as its observed value if every model assumption were correct, including the test hypothesis" (Greenland et al. 2016: 339). In other words: assuming that there is in fact no difference between the control and treatment group, the $\mathrm{p}$-value is the chance of observing the difference or a more extreme one. In addition, adequate randomization leads to difference in groups by chance. As it has already been described by Altman (1985: 126), significance tests of baseline comparisons are at best tautological because "performing a significance test to compare baseline variables is to assess the probability of something having occurred by chance when we know that it did occur by chance."

What instead of using p-values can be done to evaluate whether randomization worked properly? Schober et al. (2018) propose to use standardized differences, which measure the effect size between groups. The procedure of standard differences is straightforward and implemented in standard software packages (e.g. SAS or Stata; Yang and Dalton 2012; Bayoumi 2016). Therefore, the idea shall be outlined only briefly using the two formulas for continuous and categorical baseline variables (cf., Table 1; e.g. Austin 2009). The higher the $d$, the higher the effect size. In his effect size index, Cohen (1988) associated values of 0.2 as small, 0.5 as medium, and 0.8 as large effect sizes. ${ }^{2}$

\footnotetext{
${ }^{1}$ Altman (1985) acknowledges that p-values might be used to evaluate whether randomization is fair or not. If randomization would work perfectly then $\mathrm{H} 0$ is true (i.e., there is no difference between groups). Thus, we would expect to observe 1 statistically significant test with $\mathrm{P}<0.05$ in a total of 20 tests. We can now opposite the number of comparisons made divided through 20 , on the one hand, and the number of statistically significant comparisons, on the other one. Similar numbers indicate that there is no lack of comparability.

${ }^{2}$ It should be remarked that calclulating effect sizes of two groups on the basis of Cohen's d is only valid if one deals with two systematically-distriburted samples.
} 
Table 1 Calculating standard differences (continuous and categorical baseline)

\begin{tabular}{|c|c|c|}
\hline \multicolumn{2}{|c|}{ Continuous variables } & \multirow{2}{*}{$\begin{array}{l}\text { Dichotomous variables } \\
\qquad d=\frac{\left(\hat{p}_{T}-\hat{p}_{C}\right)}{\sqrt{\frac{\hat{p}_{T}\left(1-\hat{p}_{T}\right)+\hat{p}_{C}\left(1-\hat{p}_{C}\right)}{2}}}\end{array}$} \\
\hline & $d=\frac{\left(\bar{x}_{T}-\bar{x}_{c}\right)}{\sqrt{\frac{s_{T}^{2}+s_{C}^{2}}{2}}}$ & \\
\hline $\bar{x}_{T}\left(\bar{x}_{c}\right)$ & $\begin{array}{l}\text { Sample mean of covariate, treated } \\
\text { subjects (untreated subjects) }\end{array}$ & $\begin{array}{l}\text { Prevalence/mean of the covariate, } \\
\text { treated subjects (untreated subjects) }\end{array}$ \\
\hline$s_{T}^{2}\left(S_{C}^{2}\right)$ & $\begin{array}{l}\text { Sample variance of covariate, } \\
\text { treated subjects (untreated sub- } \\
\text { jects) }\end{array}$ & \\
\hline
\end{tabular}

\section{Systematic differences across groups}

While the last chapter dealt deviations in randomization by chance, we now want to think about whether there are scenarios in which systematic deviations can occur. The experimenter has several options in which venue the experiment can be carried out. These include the classroom, the laboratory, and the Internet. Participants in controlled economic experiments are often referred to as "volunteer subjects" because they decide on their own whether to attend an experiment (e.g. Rosenthal and Rosnow 1969). For example, the share of people who refuse to participate in classroom experiments is relatively low. Eckel and Grossman (2000) therefore speak of "pseudo-volunteers." A possible reason for this is a certain degree of social control due to the lack of anonymity. Internet-based experiments, on the other hand, exhibit a high degree of anonymity and presumably also a considerably extent of. Adequate randomization should, however, ensure that comparable groups are created within a venue; while differences in level across the venues are conceivable.

However, systematic differences between groups may also arise despite randomization. Let us assume that the experimenter is using an Internet-based experiment to investigate the behavioral influence of financial incentives. The following scenarios are examined: (1) no financial incentives, (2) medium financial incentives, and (3) high financial incentives. Each individual is randomly assigned to a scenario. The participants in the experiment experience of course the conditions to which they were assigned to. It is possible that an individual cancels participation if the monetary payoffs are considered too low and the intrinsic motivation is not sufficient to compensate for. For example, an individual could have been assigned to scenario 1 and refuses to participate. But the same individual could have attented the experiment if it had been assigned to one of the other scenarios. Similar to observation studies, econometric regressions can now be used to control for known confounding effects.

By choosing the venue, the experimenter exerts direct or indirect influence on the Individuals' self-selection decisions. The choice of venue depends upon the research question as well as considerations of advantages and disadvantages by the experimenter. For example, Internet-based experiments are related to less control than lab experiments, but the Internet makes it easier, for example, to recruit non-students. In any case, there is no free lunch. 


\section{Recommendation}

Resorting to $\mathrm{p}$-values is an inadequate, but widely spread procedure to assess whether randomization was successfull. Instead, it is better to use standardized differences. Depending on the venue of the experiment, the influence of self-selection is of varying importance. Challenges to evaluate the quality of randomization appear if self-selection is related to treatments. The experimenter must carefully keep this in mind when deciding about the venue of the experiment.

\section{References}

Altman, D.G. (1985): Comparability of Randomised Groups. The Statistician 34(1): 125-136.

Austin, P.C. (2009): Balance diagnostics for comparing the distribution of baseline covariates between treatment groups in propensity-score matched samples. Statistics in Medicine 28(25): 3083-3107.

Bayoumi, A.M. (2016): STDDIFF: Stata module to compute Standardized differences for continuous and categorical variables. Statistical Software Components S458275, Boston College Department of Economics.

Borenstein, M., Hedges, L.V., Higgins, J.P.T., Rothstein, H.R. (2009): Introduction to meta-analysis. Chichester, England: Wiley.

Cohen J. (1988): Statistical Power Analysis for the Behavioral Sciences. Hillsdale, NJ: Lawrence Erlbaum Associates Publishers.

Deaton, A., Cartwright, N. (2018): Understanding and misunderstanding randomized controlled trials. Social Science \& Medicine 210: 2-21.

Dunning, T. (2012): Natural Experiments in the Social Sciences. A DesignBased Approach. New York: Cambridge University Press.

Eckel, C.C., Grossman, P.J. (2000): Volunteers and pseudo-volunteers: The effect of recruitment method in dictator experiments. Experimental Economics 3(2): 107-120.

Greenland, S., Senn, S.J., Rothman, K.J., Carlin, J.B., Poole, C., Goodman, S.N., Altman, D.G. (2016): Statistical tests, $\mathrm{P}$ values, confidence intervals, and power: a guide to misinterpretations. European Journal of Epidemiology 31(4): 337-350.

Rosenthal, R.W., Rosnow, R.L. (1969): Artifact in Behavioral Research. New York: Academic Press.

Schober, P., Bossers, S.M., Schwarte, L.A. (2018): Statistical Significance Versus Clinical Importance of Observed Effect Sizes: What Do P Values and Confidence Intervals Really Represent? Anesthesia \& Analgesia 126(3): 1068-1072.

Yang, D., Dalton, J.E. (2012): A unified approach to measuring the effect size between two groups using SAS. SAS Global Forum 2012: Statistics and Data Analysis, Paper 335-2012. http://support.sas.com/resources/papers/proceedings12/335-2012.pdf 